Supplementary Information

\title{
Highly efficient cuprous complexes with thermally activated delayed fluorescence for solution processed organic light-emitting device
}

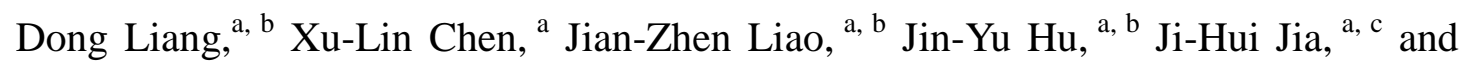
Can-Zhong $\mathrm{Lu}^{*^{\mathrm{a}}}$

${ }^{\text {a }}$ Key Laboratory of Design and Assembly of Functional Nanostructures, Fujian Institute of Research on the Structure of Matter, Chinese Academy of Sciences, Fuzhou, Fujian, 350002, P. R. China.E-mail: czlu@fjirsm.ac.cn

${ }^{\mathrm{b}}$ University of Chinese Academy of Sciences, Beijing, 100049, China

${ }^{\mathrm{c}}$ College of Materials Science and Engineering, Fujian Normal University, Fuzhou, Fujian, 350007, P. R. China. 

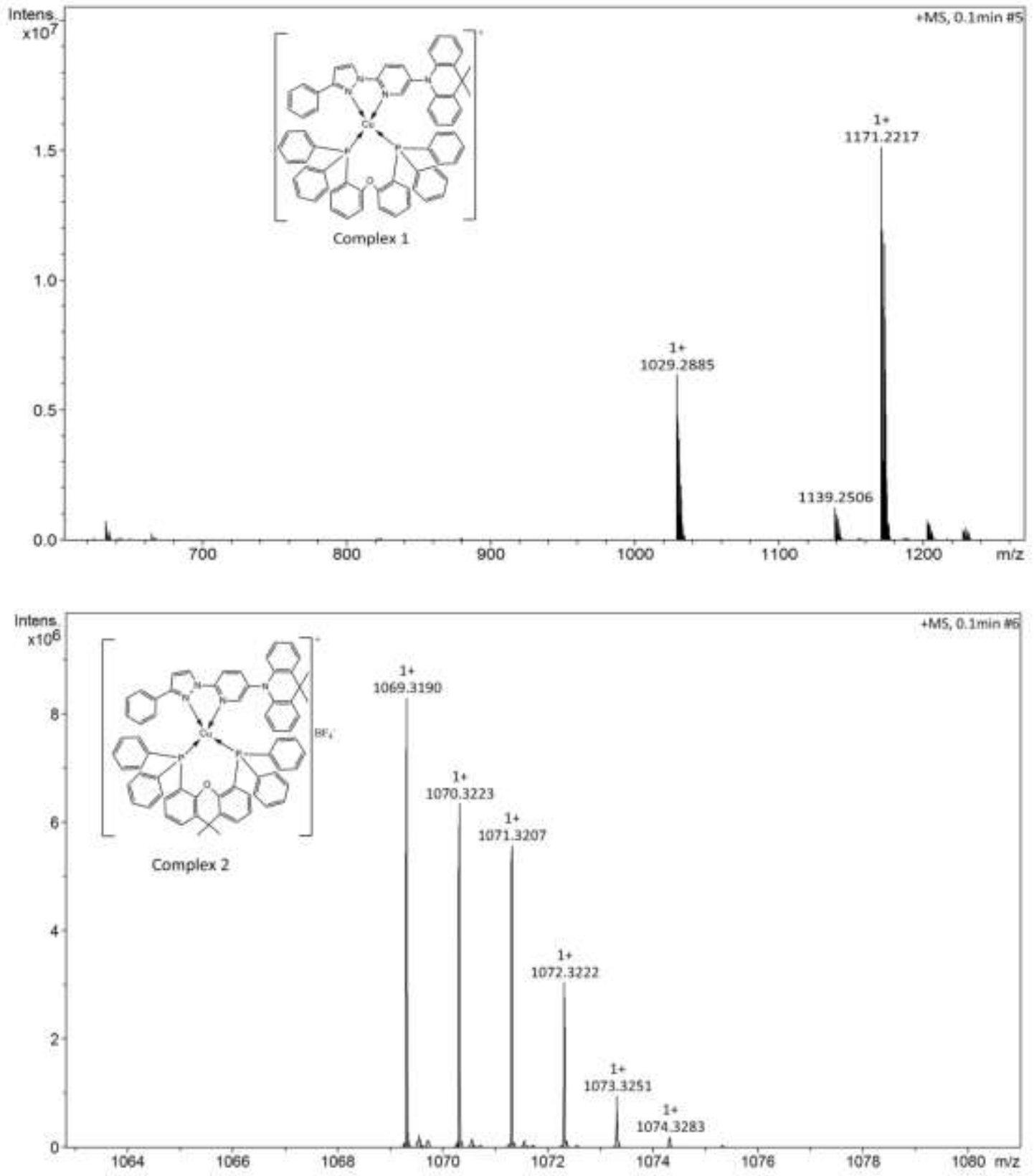

Figure $\mathbf{S 1}$ Mass spectra of complexes $\mathbf{1}$ and $\mathbf{2}$ in $\mathrm{CH}_{2} \mathrm{Cl}_{2}$. Before test, these solution samples had been exposed to air for 10 days and added $\mathrm{CH}_{2} \mathrm{Cl}_{2}$ during this period. 


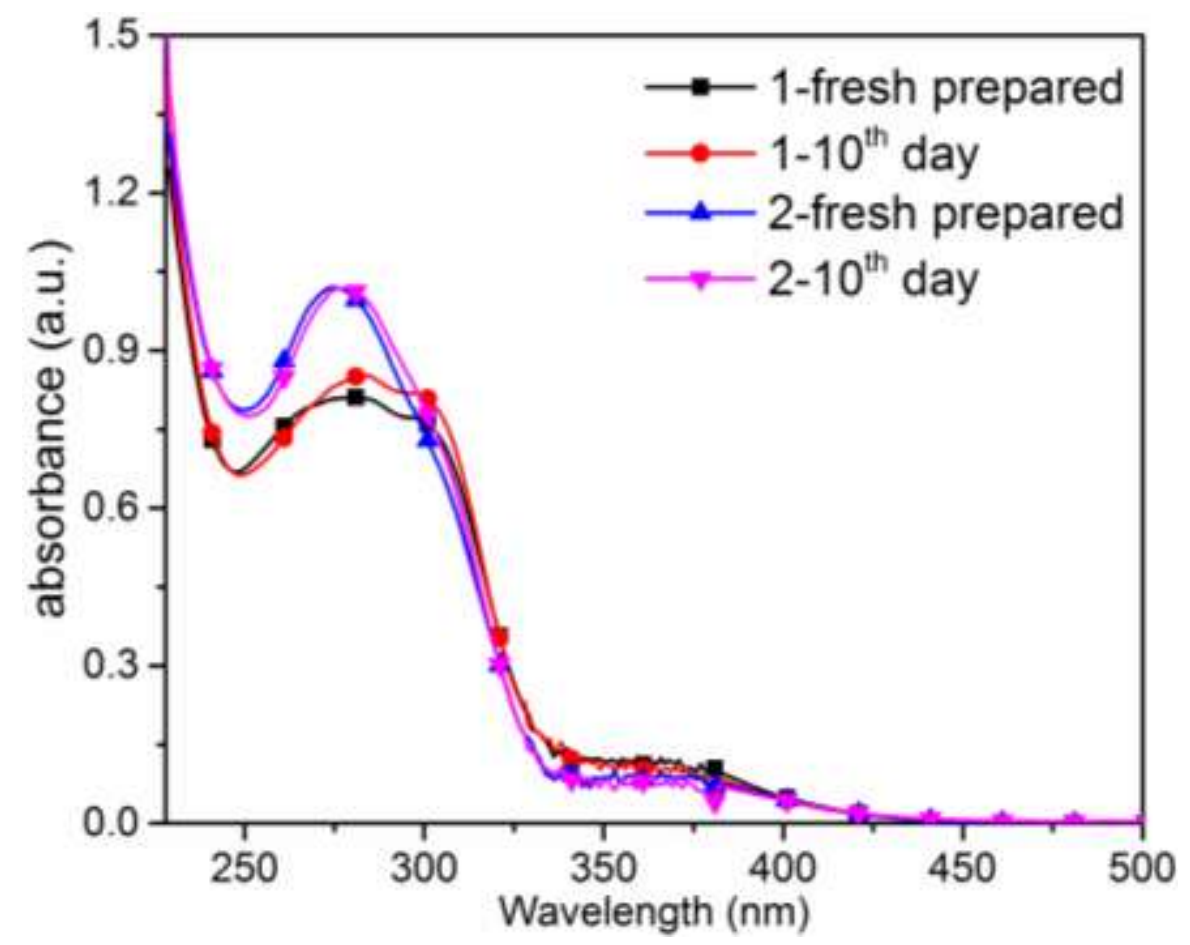

Figure S2 UV-absorption spectra of complexes $\mathbf{1}$ and $\mathbf{2}$ in $\mathrm{CH}_{2} \mathrm{Cl}_{2}$. The solution samples are tested fresh prepared and after being exposed to air for 10 days, respectively. $\mathrm{CH}_{2} \mathrm{Cl}_{2}$ was added to maintain concentration during this period. 


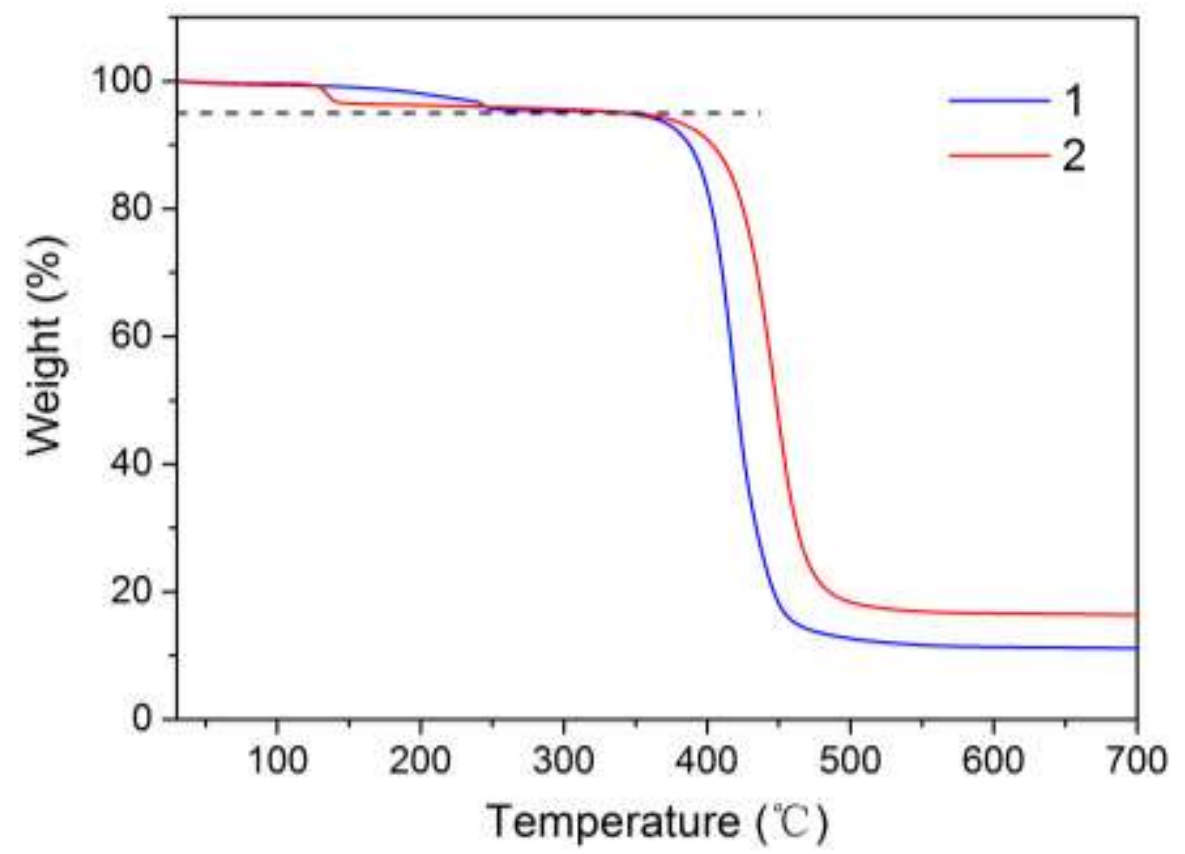

Figure S3 TGA-plots of complexes 1 and 2. The black dashed line marks $95 \%$ of the original sample weight. 
Table S1 Crystal data and structure refinement for the cuprous complexes

\begin{tabular}{|c|c|c|}
\hline Complex & 1 & 2 \\
\hline Empirical formula & $\mathrm{C}_{65} \mathrm{H}_{52} \mathrm{BCuF}_{4} \mathrm{~N}_{4} \mathrm{OP}_{2} \cdot 0.5\left(\mathrm{H}_{2} \mathrm{O}\right) \cdot 0.5\left(\mathrm{CH}_{2} \mathrm{Cl}_{2}\right)$ & $\mathrm{C}_{68} \mathrm{H}_{56} \mathrm{BCuF}_{4} \mathrm{~N}_{4} \mathrm{OP}_{2}$ \\
\hline Formula weight & 1168.865 & 1157.46 \\
\hline Crystal system & Monoclinic & Monoclinic \\
\hline Space group & P21 & $\mathrm{P} 21 / \mathrm{n}$ \\
\hline a (̊̊) & $13.2087(3)$ & $10.6932(2)$ \\
\hline$b(\AA ̊)$ & $20.7679(6)$ & $17.2639(3)$ \\
\hline$c(\AA)$ & $20.2938(5)$ & $30.7265(5)$ \\
\hline$\alpha$ (deg) & 90.00 & 90.00 \\
\hline$\beta$ (deg) & $94.776(2)$ & $96.8460(10)$ \\
\hline$\nu$ (deg) & 90.00 & 90.00 \\
\hline$V\left(\AA^{3}\right)$ & $5547.6(2)$ & $5631.86(17)$ \\
\hline Z & 2 & 4 \\
\hline$\rho_{\text {calc }}\left(\mathrm{g} \cdot \mathrm{cm}^{-3}\right)$ & 1.399 & 1.365 \\
\hline$\mu\left(\mathrm{mm}^{-1}\right)$ & 2.060 & 1.592 \\
\hline$F(000)$ & 2416 & 2400 \\
\hline$\lambda(\AA ̊)$ & 1.54184 & 1.54184 \\
\hline Reflections collected/unique & $23666 / 16954$ & $40377 / 10322$ \\
\hline $\mathrm{R}_{\text {int }}$ & 0.0309 & 0.0285 \\
\hline$\Theta$ range (deg) & $3.36-74.41$ & $3.87-68.25$ \\
\hline GOF on $F^{2}$ & 1.037 & 1.016 \\
\hline $\mathrm{R}_{1} / \mathrm{wR}_{2} \quad[\mathrm{I} \geqslant 2 \sigma(\mathrm{I})]$ & $0.0377 / 0.0965$ & $0.0459 / 0.1246$ \\
\hline $\mathrm{R}_{1} / \mathrm{wR}_{2}$ (all data) & $0.0422 / 0.1012$ & $0.0494 / 0.1277$ \\
\hline
\end{tabular}


Table S2 Selected bond length ( $\AA$ ) and bond angles (deg) for the cuprous complexes

\begin{tabular}{ccc}
\hline Complex & 1 & 2 \\
\hline $\mathrm{Cu}-\mathrm{N}_{1}$ & $2.085(3)$ & $2.0752(19)$ \\
$\mathrm{Cu}-\mathrm{N}_{3}$ & $2.062(3)$ & $2.1161(19)$ \\
$\mathrm{Cu}-\mathrm{P}_{1}$ & $2.2715(10)$ & $2.2290(6)$ \\
$\mathrm{Cu}-\mathrm{P}_{2}$ & $2.2446(9)$ & $2.2942(6)$ \\
$\mathrm{N}_{1}-\mathrm{Cu}-\mathrm{N}_{3}$ & $79.12(11)$ & $78.31(8)$ \\
$\mathrm{P}_{1}-\mathrm{Cu}-\mathrm{P}_{2}$ & $116.34(3)$ & $121.64(2)$ \\
$\mathrm{N}_{1}-\mathrm{Cu}-\mathrm{P}_{1}$ & $115.81(9)$ & $124.46(6)$ \\
$\mathrm{N}_{1}-\mathrm{Cu}-\mathrm{P}_{2}$ & $121.90(8)$ & $103.35(5)$ \\
$\mathrm{N}_{3}-\mathrm{Cu}-\mathrm{P}_{1}$ & $104.98(9)$ & $119.67(6)$ \\
$\mathrm{N}_{3}-\mathrm{Cu}-\mathrm{P}_{2}$ & $109.82(8)$ & $99.50(6)$ \\
\hline
\end{tabular}


Table S3 Photophysical data of ligand PNNA

\begin{tabular}{ccccccc}
\hline & $\begin{array}{c}\lambda_{\text {em }} \text { in } \\
\text { degassed } \\
\text { toluene } \\
(\mathrm{nm})\end{array}$ & $\begin{array}{c}\text { PLQY in } \\
\text { degassed } \\
\text { toluene } \\
(\%)^{\text {a }}\end{array}$ & $\begin{array}{c}\text { PLQY in } \\
\text { air-equilibrated } \\
\text { toluene }(\%)^{a}\end{array}$ & $\begin{array}{c}\text { rel portion of } \\
\text { delayed } \\
\text { fluorescence } \\
\text { in degassed } \\
\text { toluene }(\%)\end{array}$ & $\begin{array}{c}\text { delayed } \\
\text { fluorescence } \\
\text { lifetime in } \\
\text { degassed }\end{array}$ & $\begin{array}{c}\text { prompt } \\
\text { fluorescence } \\
\text { lifetime in }\end{array}$ \\
\hline PNNA & 420 & 76 & 29 & 62 & 58 & $\begin{array}{c}\text { degassed } \\
\text { toluene }(\mathrm{ns})\end{array}$ \\
\hline
\end{tabular}




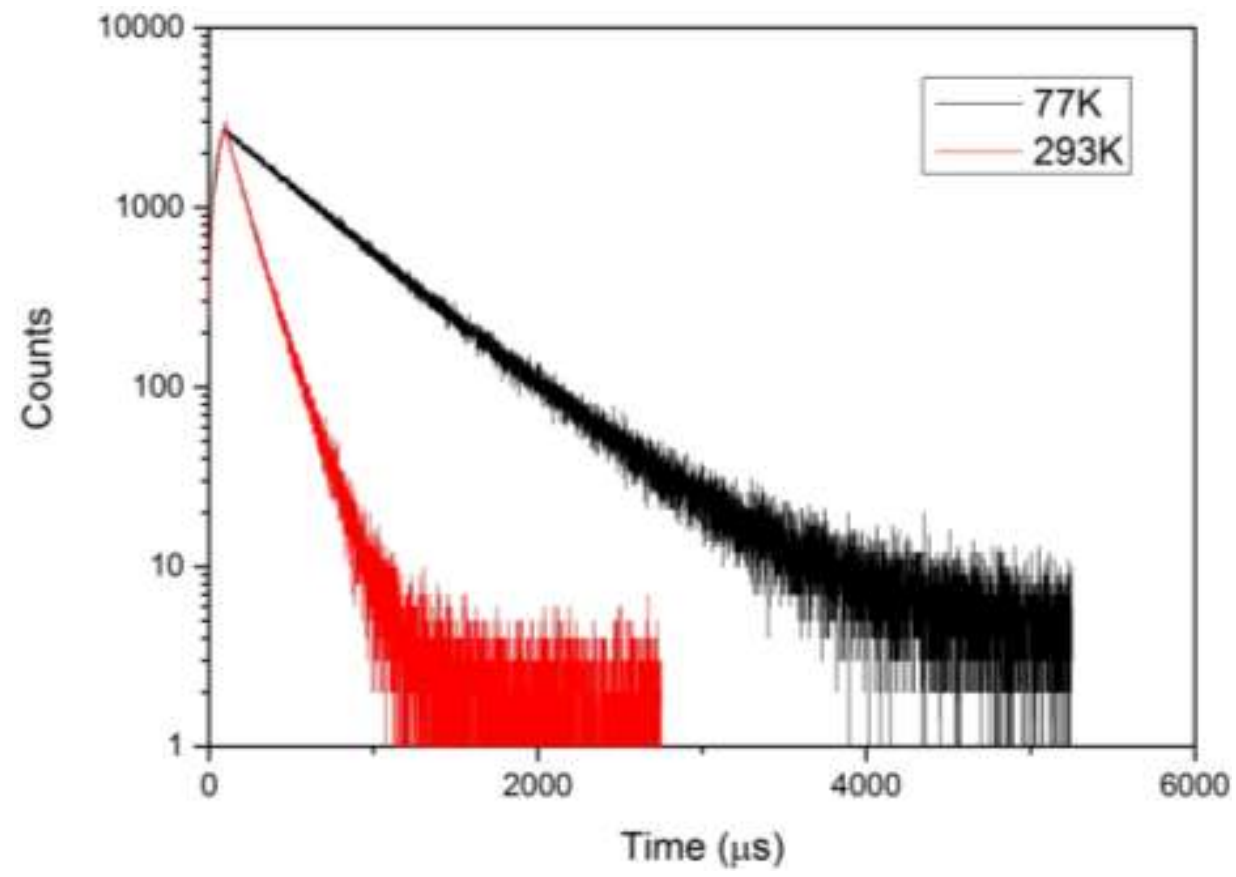

Figgure S4 Transient decay spectra of 1 at 77k and 293k 


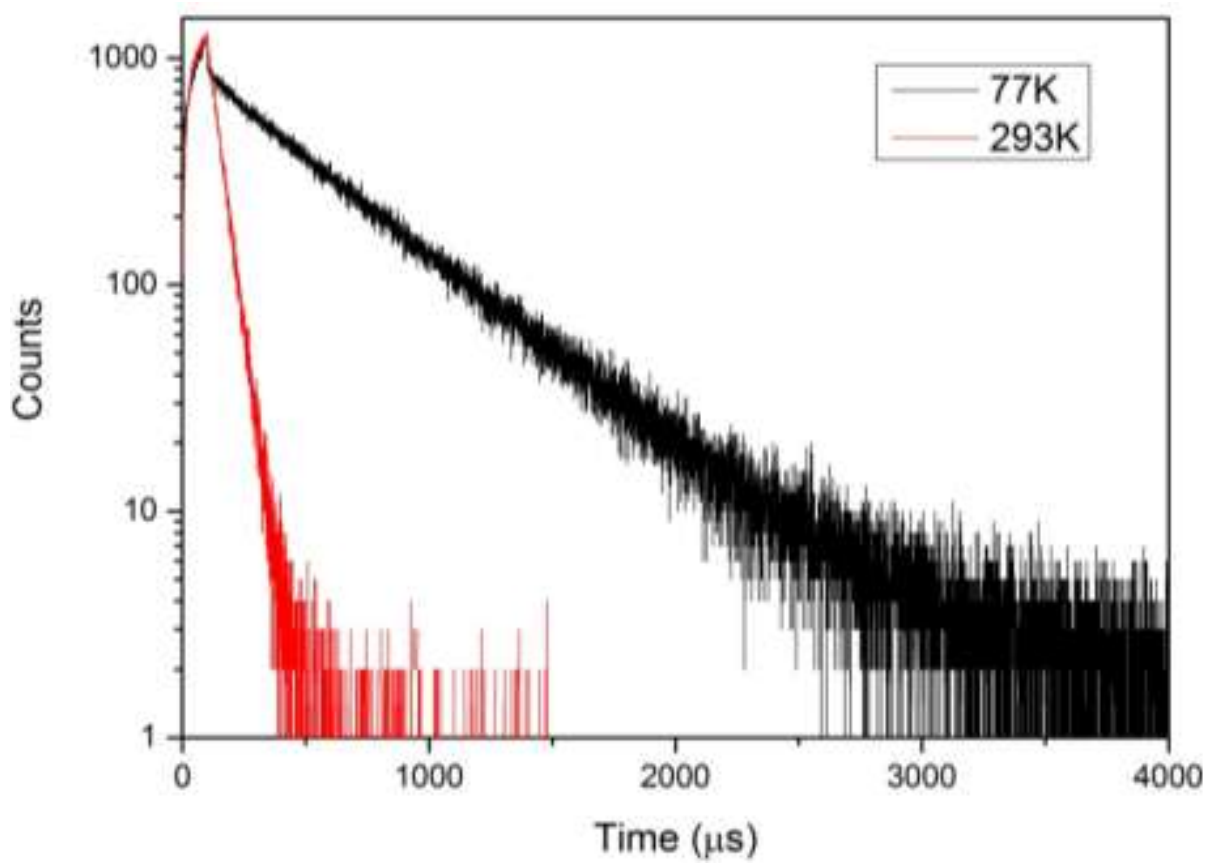

Figgure S5 Transient decay spectra of $\mathbf{2}$ at 77k and 293k 
Table S4 Detailed data for device optimization

\begin{tabular}{|c|c|c|c|c|c|c|}
\hline & \multirow{2}{*}{ complex } & & EQE (\%) & $\mathrm{CE}(\mathrm{cd} / \mathrm{A})$ & $\mathrm{V}_{\text {on }}(\mathrm{V})$ & $\mathrm{B}_{\max }$ \\
\hline & & & Max (@100cd/m²) & $\operatorname{Max}\left(@ 100 c d / m^{2}\right)$ & $@ 1 \mathrm{~cd} / \mathrm{m}^{2}$ & $\left(\mathrm{~cd} / \mathrm{m}^{2}\right)$ \\
\hline \multirow{6}{*}{1} & \multirow{3}{*}{$\begin{array}{c}\text { doping } \\
\text { concentration }\end{array}$} & $10 \%$ & $5.27(4.55)$ & $12.98(11.22)$ & 7.7 & 3055 \\
\hline & & $30 \%$ & $3.29(3.28)$ & 8.69 (8.67) & 6.4 & 2626 \\
\hline & & $100 \%$ & 1.18 (1.05) & $3.69(3.28)$ & 5.6 & 1375 \\
\hline & thickness of & $40 \mathrm{~nm}$ & $4.57(4.52)$ & $11.48(11.36)$ & 5.7 & 4617 \\
\hline & 3TPYMB layer & $60 \mathrm{~nm}$ & 7.07 (7.03) & $18.72(18.62)$ & 6.5 & 4012 \\
\hline & $\begin{array}{l}\text { thickness of } \\
\text { emitting layer }\end{array}$ & $30 \mathrm{~nm}$ & $6.58(6.58)$ & $16.20(16.20)$ & 6.6 & 5334 \\
\hline \multirow{6}{*}{2} & & $10 \%$ & $6.71(6.58)$ & 17.59 (17.27) & 7.7 & 4223 \\
\hline & & $30 \%$ & $6.98(6.78)$ & $19.24(18.68)$ & 6.2 & 6567 \\
\hline & & $100 \%$ & $1.11(0.80)$ & $3.48(2.51)$ & 5.9 & 1021 \\
\hline & thickness of & $40 \mathrm{~nm}$ & $6.29(6.05)$ & $16.85(16.23)$ & 6.7 & 5032 \\
\hline & 3TPYMB layer & $60 \mathrm{~nm}$ & $6.32(6.06)$ & 16.66 (15.99) & 7.5 & 3396 \\
\hline & $\begin{array}{l}\text { thickness of } \\
\text { emitting layer }\end{array}$ & $30 \mathrm{~nm}$ & $5.11(5.11)$ & 13.97 (13.97) & 6.7 & 3594 \\
\hline
\end{tabular}


Table S5 Performance comparison with the representative analogous complexes

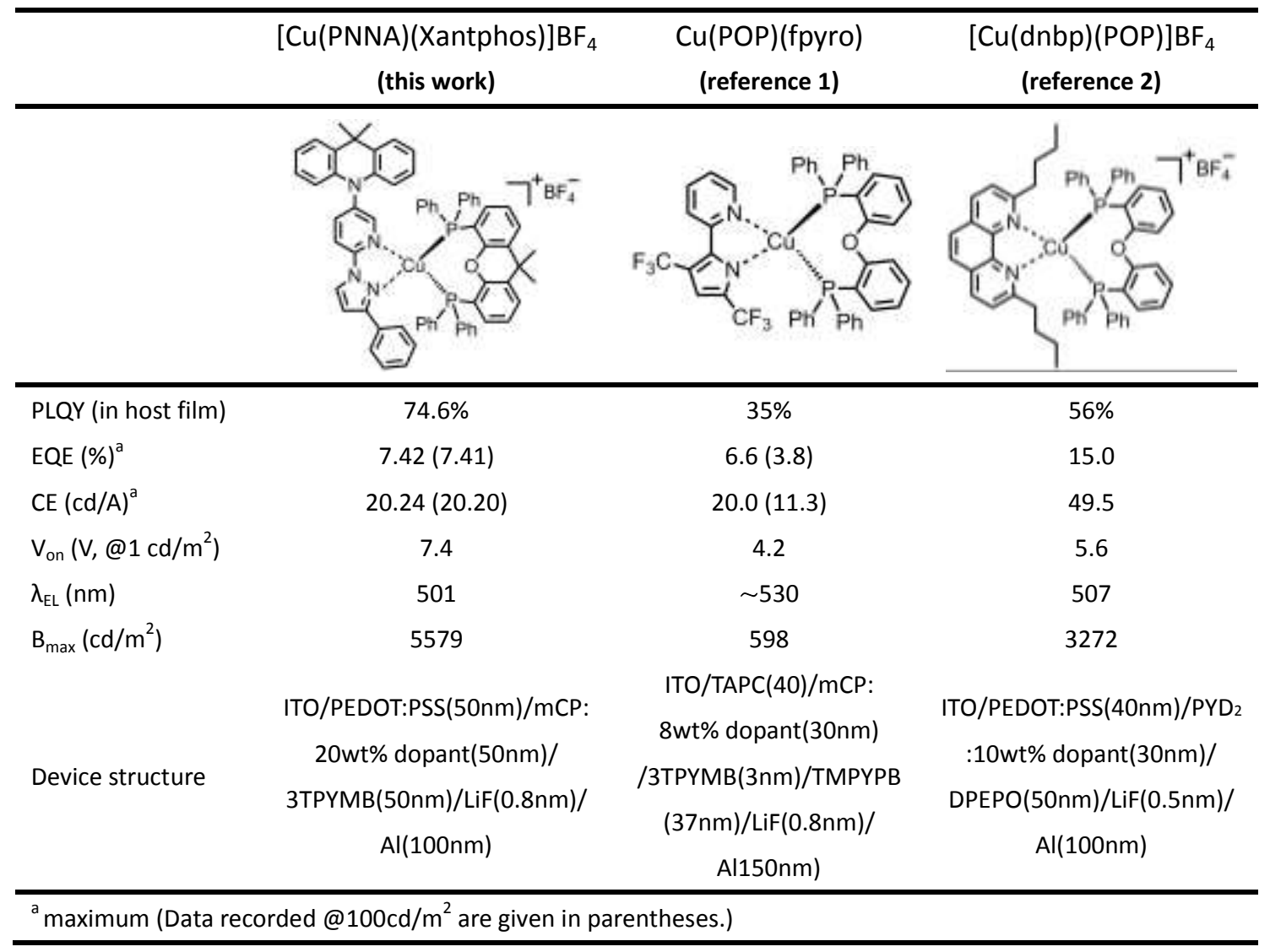

\section{References:}

(1). Hsu, C. W.; Lin, C. C.; Chung, M. W.; Chi, Y.; Lee, G. H.; Chou, P. T.; Chang, C. H.; Chen, P. Y., J Am Chem. Soc. 2011, 133, 12085.

(2). Zhang, Q. S.; Komino, T.; Huang, S. P.; Matsunami, S.; Goushi, K.; Adachi, C., Adv. Funct. Mater. 2012, 22, 2327. 